Supporting Information for:

\title{
New Insights into the Excited State Dynamics of Quinoline-Pyrazole Isomerism
}

Zhe Tanga, Panwang Zhou ${ }^{\mathrm{a}^{*}}$

a Institute of Molecular Sciences and Engineering, Institute of Frontier and Interdisciplinary Science, Shandong University, Qingdao 266237, P.R. China

E-mail: pwzhou@sdu.edu.cn Tel: +86-532-58632158

\section{Contents}

Table S1 S2

Table S2 S2

Table S3 S2

Figure S1 .S3

Figure S2 .S3

Optimized Geometries S4-S15 
Table S1. Comparing theoretical absorption and emission energies (in $\mathrm{nm}$ ) for QP-1, QP-2, PT-1 and PT-2 in CYH solvents based on different functionals (CAM-B3LYP, B3LYP, WB97XD, PBE0) and the experimentally measured values.

\begin{tabular}{|c|c|c|c|c|c|c|}
\hline & & $\begin{array}{c}\text { CAM-B3L } \\
\text { YP }\end{array}$ & B3LYP & WB97XD & PBE0 & exp \\
\hline \multirow{2}{*}{ QP-1 } & abs & 285 & 315 & 283 & 306 & $\sim 310$ \\
\cline { 2 - 7 } & emission & 326 & 361 & 325 & 350 & 350 \\
\hline \multirow{2}{*}{ PT-1 } & emission & 491 & 647 & 475 & 608 & 570 \\
\hline \multirow{2}{*}{ QP-2 } & abs & 284 & 307 & 282 & 299 & $\sim 310$ \\
\cline { 2 - 7 } & emission & 331 & 357 & 328 & 348 & $\sim 340$ \\
\hline PT-2 & emission & 406 & 462 & 402 & 446 & - \\
\hline
\end{tabular}

Table S2. Relative free energy $(\mathrm{kcal} / \mathrm{mol})$ in $\mathrm{S}_{1}$ states, for QP-2, PT-2, TICT-2 and Trans-2 based on the CAM-B3LYP-D3/TZVP/IEFPCM levels.

\begin{tabular}{|c|c|c|c|c|}
\hline & QP-2 & PT-2 & TICT-2 & Trans-2 \\
\hline energy & 0 & -3.44 & -5.90 & 1.56 \\
\hline
\end{tabular}

Table S3. Energy terms needed to perform excited state energy decomposition calculated at CAM-B3LYP -D3/TZVP/IEFPCM levels.

\begin{tabular}{|c|c|c|c|c|c|c|c|}
\hline Conf. & $\begin{array}{c}\text { S0 state } \\
\text { (a.u.) }\end{array}$ & $\begin{array}{c}\text { HOMO } \\
(\mathrm{eV})\end{array}$ & $\begin{array}{c}\text { LUMO } \\
(\mathrm{eV})\end{array}$ & $\begin{array}{c}\text { H-L gap } \\
(\mathrm{eV})\end{array}$ & $\begin{array}{c}\text { FC state } \\
(\text { a.u. })\end{array}$ & $\begin{array}{c}\text { Hole-elec. } \\
(\mathrm{eV})\end{array}$ & $\begin{array}{c}\text { Relaxed } \\
\text { S1 state(a.u. })\end{array}$ \\
\hline PT-1 & -963.9027 & -7.3933 & -1.8830 & 5.5103 & -963.7863 & -2.3429 & -963.7979 \\
\hline TICT-1 & -963.8820 & -7.6346 & -2.1606 & 5.4740 & -963.7594 & -2.1379 & -963.7791 \\
\hline PT-2 & -963.9127 & -7.7634 & -1.8803 & 5.8831 & -963.7861 & -2.4381 & -963.7932 \\
\hline TICT-2 & -963.8864 & -7.7444 & -2.2341 & 5.5103 & -963.7777 & -2.5524 & -963.7956 \\
\hline
\end{tabular}


Figure S1. Optimized QP-3 geometric in $\mathrm{S}_{0}$ and $\mathrm{S}_{1}$ states based on CAM-B3LYP-D3/TZVP/IEFPCM levels.

$\mathbf{S}_{0}$

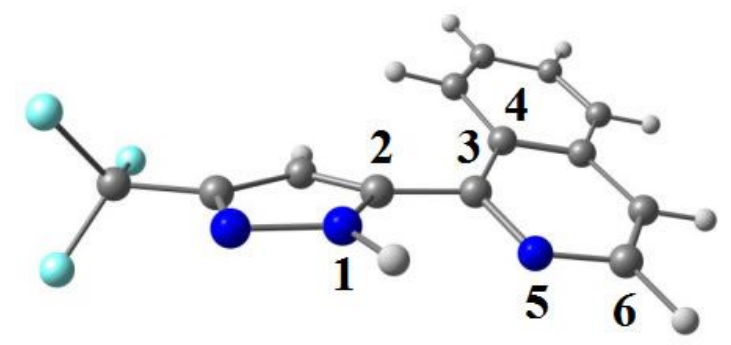

Dihedral $(\mathrm{N} 1-\mathrm{C} 2-\mathrm{C} 3-\mathrm{N} 5)=22.9^{\circ}$

Dihedral (C4-C3-N5-C6) $=-2.8^{\circ}$
$\mathbf{S}_{1}$

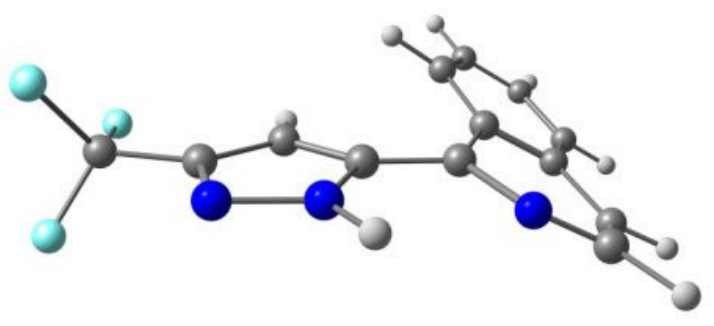

Dihedral $(\mathrm{N} 1-\mathrm{C} 2-\mathrm{C} 3-\mathrm{N} 5)=8.9^{\circ}$

Dihedral $(\mathrm{C} 4-\mathrm{C} 3-\mathrm{N} 5-\mathrm{C} 6)=-23.9^{\circ}$

Figure S2. Optimized TICT-2 and Trans-2 geometric in $\mathrm{S}_{1}$ state based on CAM-B3LYP methods.

TICT-2

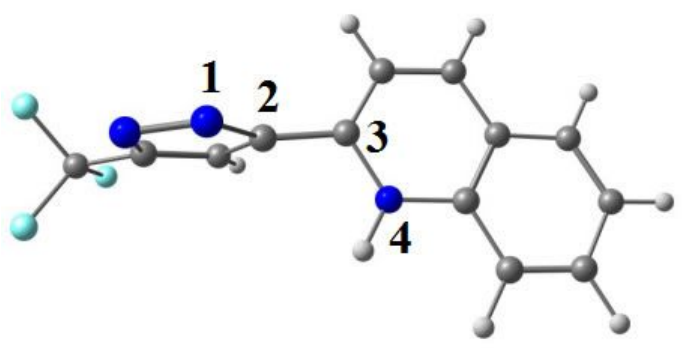

Dihedral $(\mathrm{N} 1-\mathrm{C} 2-\mathrm{C} 3-\mathrm{N} 4)=89.5^{\circ}$
Trans-2

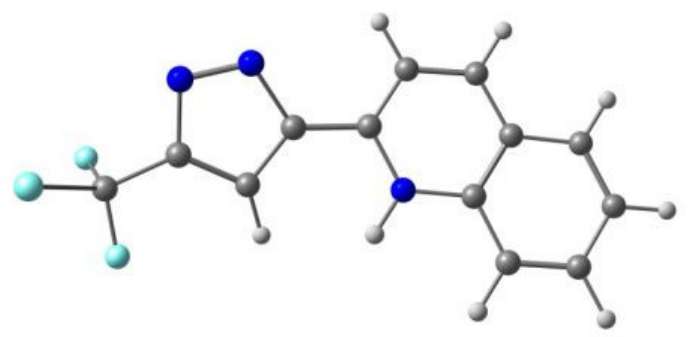

Dihedral $(\mathrm{N} 1-\mathrm{C} 2-\mathrm{C} 3-\mathrm{N} 4)=159.2^{\circ}$ 
$\mathrm{S}_{0}$ geometry of QP-1 in cyclohexane solvent, cam-b3lyp/tzvp/iefpcm, empiricaldispersion $=\mathrm{gd} 3 \mathrm{bj}$

Energy $=-963.785245$

\begin{tabular}{|c|c|c|c|}
\hline $\mathrm{C}$ & -5.65531200 & -0.35676900 & 0.00018300 \\
\hline $\mathrm{C}$ & -4.86909000 & 0.75841700 & 0.00014200 \\
\hline $\mathrm{C}$ & -3.46377300 & 0.63153900 & 0.00005800 \\
\hline $\mathrm{C}$ & -2.87312600 & -0.65177100 & 0.00002400 \\
\hline $\mathrm{C}$ & -3.71172000 & -1.78959100 & 0.00010100 \\
\hline $\mathrm{C}$ & -5.06823800 & -1.64016800 & 0.00016800 \\
\hline $\mathrm{H}$ & -3.02437700 & 2.74922100 & 0.00030300 \\
\hline $\mathrm{H}$ & -6.73326200 & -0.26419400 & 0.00022700 \\
\hline $\mathrm{H}$ & -5.30960900 & 1.74791100 & 0.00016000 \\
\hline $\mathrm{C}$ & -2.59745600 & 1.75056600 & 0.00001500 \\
\hline $\mathrm{C}$ & -1.46489200 & -0.72530500 & -0.00009700 \\
\hline $\mathrm{H}$ & -3.26385600 & -2.77532600 & 0.00009000 \\
\hline $\mathrm{H}$ & -5.70679600 & -2.51407700 & 0.00021900 \\
\hline $\mathrm{C}$ & -0.73039600 & 0.42664100 & -0.00025100 \\
\hline $\mathrm{H}$ & -0.97720300 & -1.69126800 & 0.00011400 \\
\hline $\mathrm{C}$ & 0.73069500 & 0.42610900 & -0.00041500 \\
\hline $\mathrm{C}$ & 1.67771700 & -0.57583600 & -0.00114200 \\
\hline $\mathrm{N}$ & 1.42145300 & 1.58783900 & 0.00028100 \\
\hline $\mathrm{C}$ & 2.89825700 & 0.11069100 & -0.00102600 \\
\hline $\mathrm{H}$ & 1.52316500 & -1.63963500 & -0.00201800 \\
\hline $\mathrm{H}$ & 1.00840100 & 2.50833100 & 0.00028300 \\
\hline $\mathrm{N}$ & 2.73813700 & 1.42240700 & -0.00014900 \\
\hline $\mathrm{C}$ & 4.27007500 & -0.47044200 & 0.00000100 \\
\hline $\mathrm{F}$ & 4.21178600 & -1.81486800 & -0.01510600 \\
\hline $\mathrm{F}$ & 4.98913000 & -0.09225000 & -1.06934100 \\
\hline $\mathrm{F}$ & 4.97697300 & $\begin{array}{l}-0.11653100 \\
\text { S4 }\end{array}$ & 1.08598000 \\
\hline
\end{tabular}


$\mathrm{N}$

$-1.29872000$

1.66509400

$-0.00009500$

$\mathrm{S}_{0}$ geometry of PT-1 in cyclohexane solvent, cam-b3lyp/tzvp/iefpcm, empiricaldispersion $=\mathrm{gd} 3 \mathrm{bj}$

Energy $=-963.753069$

\begin{tabular}{|c|c|c|c|}
\hline $\mathrm{C}$ & -5.66671100 & 0.26594300 & -0.00120600 \\
\hline $\mathrm{C}$ & -4.85069700 & -0.81951000 & -0.00218300 \\
\hline $\mathrm{C}$ & -3.44293200 & -0.64376100 & -0.00108200 \\
\hline $\mathrm{C}$ & -2.88161200 & 0.66632100 & 0.00068300 \\
\hline $\mathrm{C}$ & -3.76878100 & 1.77189800 & 0.00179700 \\
\hline $\mathrm{C}$ & -5.11471800 & 1.57160300 & 0.00092100 \\
\hline $\mathrm{H}$ & -2.90647200 & -2.74854500 & -0.00261300 \\
\hline $\mathrm{H}$ & -6.74097100 & 0.14258400 & -0.00200700 \\
\hline $\mathrm{H}$ & -5.25632100 & -1.82318500 & -0.00376600 \\
\hline $\mathrm{C}$ & -2.56660500 & -1.72204100 & -0.00148000 \\
\hline $\mathrm{C}$ & -1.48785100 & 0.81484000 & 0.00146800 \\
\hline $\mathrm{H}$ & -3.35735300 & 2.77267400 & 0.00338800 \\
\hline $\mathrm{H}$ & -5.78257400 & 2.42318300 & 0.00185800 \\
\hline $\mathrm{C}$ & -0.66677800 & -0.28520900 & 0.00075700 \\
\hline $\mathrm{H}$ & -1.04675500 & 1.80132200 & 0.00292000 \\
\hline $\mathrm{C}$ & 0.76625000 & -0.33554000 & 0.00136300 \\
\hline $\mathrm{C}$ & 1.74328300 & 0.65810100 & -0.00158900 \\
\hline $\mathrm{N}$ & 1.34475200 & -1.56088300 & 0.00431900 \\
\hline $\mathrm{C}$ & 2.90903900 & -0.08750300 & -0.00036600 \\
\hline $\mathrm{H}$ & 1.63661500 & 1.72894500 & -0.00489400 \\
\hline $\mathrm{H}$ & -0.58062700 & -2.28724400 & -0.00033700 \\
\hline $\mathrm{N}$ & 2.65359000 & -1.41054800 & 0.00345500 \\
\hline $\mathrm{C}$ & 4.30538500 & 0.40657400 & -0.00112300 \\
\hline $\mathrm{F}$ & 4.34154500 & 1.75729800 & -0.02081600 \\
\hline $\mathrm{F}$ & 5.00342700 & 0.01888500 & 1.08544900 \\
\hline $\mathrm{F}$ & 5.01355100 & $\begin{array}{l}-0.01374500 \\
\text { S5 }\end{array}$ & -1.06831900 \\
\hline
\end{tabular}


$\begin{array}{llll}\mathrm{N} & -1.26346700 & -1.52027900 & -0.00050800\end{array}$

$\mathrm{S}_{1}$ geometry of QP-1 in cyclohexane solvent, cam-b3lyp/tzvp/iefpcm, empiricaldispersion $=\mathrm{gd} 3 \mathrm{bj}$

Energy $=-963.642772$

\begin{tabular}{|c|c|c|c|}
\hline $\mathrm{C}$ & 5.64290000 & 0.44092500 & 0.00012500 \\
\hline $\mathrm{C}$ & 4.84073400 & -0.72943400 & 0.00008100 \\
\hline $\mathrm{C}$ & 3.45578500 & -0.64564300 & 0.00003700 \\
\hline $\mathrm{C}$ & 2.84757300 & 0.65705600 & -0.00004600 \\
\hline $\mathrm{C}$ & 3.66438100 & 1.79865300 & 0.00001900 \\
\hline $\mathrm{C}$ & 5.06786800 & 1.68782200 & 0.00009700 \\
\hline $\mathrm{H}$ & 3.05384800 & -2.77181900 & 0.00009200 \\
\hline $\mathrm{H}$ & 6.72007100 & 0.33655700 & 0.00017900 \\
\hline $\mathrm{H}$ & 5.31762400 & -1.70146000 & 0.00011100 \\
\hline $\mathrm{C}$ & 2.60872900 & -1.78390200 & 0.00005100 \\
\hline $\mathrm{C}$ & 1.45061200 & 0.69647700 & -0.00017300 \\
\hline $\mathrm{H}$ & 3.19811300 & 2.77599700 & -0.00000900 \\
\hline $\mathrm{H}$ & 5.68082700 & 2.57807000 & 0.00015000 \\
\hline $\mathrm{C}$ & 0.71156600 & -0.52715400 & -0.00012200 \\
\hline $\mathrm{H}$ & 0.92747300 & 1.64266200 & -0.00016800 \\
\hline $\mathrm{C}$ & -0.72117000 & -0.49755700 & -0.00005800 \\
\hline $\mathrm{C}$ & -1.64874300 & 0.54052000 & -0.00082400 \\
\hline $\mathrm{N}$ & -1.45433600 & -1.63995800 & 0.00059900 \\
\hline $\mathrm{C}$ & -2.88246800 & -0.10632700 & -0.00082700 \\
\hline $\mathrm{H}$ & -1.46027800 & 1.59853000 & -0.00174700 \\
\hline $\mathrm{H}$ & -1.06765900 & -2.57307200 & 0.00096200 \\
\hline $\mathrm{N}$ & -2.75557500 & -1.42880400 & 0.00022300 \\
\hline $\mathrm{C}$ & -4.24080500 & 0.50889600 & -0.00017500 \\
\hline $\mathrm{F}$ & -4.14575200 & 1.84963300 & -0.01407200 \\
\hline $\mathrm{F}$ & -4.96389500 & 0.14685400 & -1.07049000 \\
\hline $\mathrm{F}$ & -4.95268000 & $\begin{array}{l}0.16899600 \\
\text { S6 }\end{array}$ & 1.08516200 \\
\hline
\end{tabular}


$\mathrm{N}$

$1.26836200 \quad-1.73363800$

0.00002300

$\mathrm{S}_{1}$ geometry of PT-1 in cyclohexane solvent, cam-b3lyp/tzvp/iefpcm, empiricaldispersion $=\mathrm{gd} 3 \mathrm{bj}$

Energy $=-963.651891$

\begin{tabular}{|c|c|c|c|}
\hline $\mathrm{C}$ & -5.64065900 & 0.39139700 & 0.00000000 \\
\hline $\mathrm{C}$ & -4.84894400 & -0.74837700 & 0.00001700 \\
\hline $\mathrm{C}$ & -3.45068500 & -0.64526600 & 0.00000400 \\
\hline $\mathrm{C}$ & -2.86788400 & 0.66243800 & -0.00002600 \\
\hline $\mathrm{C}$ & -3.68140100 & 1.77983000 & -0.00004300 \\
\hline $\mathrm{C}$ & -5.07326700 & 1.65639800 & -0.00003000 \\
\hline $\mathrm{H}$ & -2.99867500 & -2.77804100 & 0.00004800 \\
\hline $\mathrm{H}$ & -6.71795700 & 0.28519900 & 0.00001000 \\
\hline $\mathrm{H}$ & -5.30934700 & -1.72820700 & 0.00004100 \\
\hline $\mathrm{C}$ & -2.61172200 & -1.77127000 & 0.00002100 \\
\hline $\mathrm{C}$ & -1.42940400 & 0.73942000 & -0.00004200 \\
\hline $\mathrm{H}$ & -3.22604200 & 2.76261700 & -0.00006700 \\
\hline $\mathrm{H}$ & -5.69806900 & 2.53822200 & -0.00004400 \\
\hline $\mathrm{C}$ & -0.66945700 & -0.38659300 & -0.00001900 \\
\hline $\mathrm{H}$ & -0.94435600 & 1.70499900 & -0.00006500 \\
\hline $\mathrm{C}$ & 0.75426100 & -0.41666300 & -0.00002800 \\
\hline $\mathrm{C}$ & 1.71956100 & 0.62056300 & -0.00000800 \\
\hline $\mathrm{N}$ & 1.40722000 & -1.64359500 & -0.00004300 \\
\hline $\mathrm{C}$ & 2.88954700 & -0.06076300 & -0.00001300 \\
\hline $\mathrm{H}$ & 1.56360300 & 1.68416400 & 0.00000800 \\
\hline $\mathrm{H}$ & -0.64340000 & -2.43430600 & 0.00001700 \\
\hline $\mathrm{N}$ & 2.67181200 & -1.44119600 & -0.00003300 \\
\hline $\mathrm{C}$ & 4.28101200 & 0.46211500 & 0.00003200 \\
\hline $\mathrm{F}$ & 4.28626100 & 1.80625300 & -0.00008400 \\
\hline $\mathrm{F}$ & 4.96904000 & 0.05805400 & 1.07892700 \\
\hline $\mathrm{F}$ & 4.96918700 & $\begin{array}{l}0.05786000 \\
\text { S7 }\end{array}$ & -1.07869000 \\
\hline
\end{tabular}


$\mathrm{N}$

$\begin{array}{lll}-1.26073200 & -1.63428500 & 0.00000300\end{array}$

$\mathrm{S}_{0}$ geometry of QP-2 in cyclohexane solvent, cam-b3lyp/tzvp/iefpcm, empiricaldispersion $=\mathrm{gd} 3 \mathrm{bj}$

Energy $=-963.786952$

\begin{tabular}{|c|c|c|c|}
\hline $\mathrm{C}$ & 5.13268200 & -1.36299200 & 0.00023900 \\
\hline $\mathrm{C}$ & 3.78433900 & -1.57157700 & -0.00018500 \\
\hline $\mathrm{C}$ & 2.89478600 & -0.47414300 & -0.00011900 \\
\hline $\mathrm{C}$ & 3.42154200 & 0.84001100 & 0.00033700 \\
\hline $\mathrm{C}$ & 4.82154500 & 1.02393600 & 0.00082000 \\
\hline $\mathrm{C}$ & 5.65843900 & -0.05381400 & 0.00077500 \\
\hline $\mathrm{H}$ & 5.80982800 & -2.20725600 & 0.00016600 \\
\hline $\mathrm{H}$ & 3.36377000 & -2.56817700 & -0.00060200 \\
\hline $\mathrm{C}$ & 2.50099400 & 1.91085100 & 0.00027700 \\
\hline $\mathrm{H}$ & 5.21733900 & 2.03217000 & 0.00122100 \\
\hline $\mathrm{H}$ & 6.73081500 & 0.09042700 & 0.00113700 \\
\hline $\mathrm{C}$ & 1.16606600 & 1.65377700 & -0.00025200 \\
\hline $\mathrm{C}$ & 0.73470000 & 0.30596600 & -0.00067000 \\
\hline $\mathrm{H}$ & 2.86950100 & 2.92938400 & 0.00066900 \\
\hline $\mathrm{H}$ & 0.44110000 & 2.45525600 & -0.00021700 \\
\hline $\mathrm{C}$ & -0.69135600 & -0.02126600 & -0.00079500 \\
\hline $\mathrm{C}$ & -1.83593300 & 0.74642100 & -0.00286800 \\
\hline $\mathrm{N}$ & -1.10716600 & -1.30724600 & 0.00039500 \\
\hline $\mathrm{C}$ & -2.87468700 & -0.19323200 & -0.00299000 \\
\hline $\mathrm{H}$ & -1.92193700 & 1.81788500 & -0.00509400 \\
\hline $\mathrm{H}$ & -0.50153900 & -2.11431000 & 0.00089500 \\
\hline $\mathrm{N}$ & -2.42639600 & -1.43639000 & -0.00085300 \\
\hline $\mathrm{N}$ & 1.55938000 & -0.71212900 & -0.00054200 \\
\hline $\mathrm{C}$ & -4.34146600 & 0.06925500 & 0.00000300 \\
\hline $\mathrm{F}$ & -4.58161300 & 1.39240500 & -0.04535000 \\
\hline $\mathrm{F}$ & -4.96420500 & $\begin{array}{l}-0.48504300 \\
\text { s8 }\end{array}$ & -1.05201200 \\
\hline
\end{tabular}




$$
\text { F } \quad-4.94523800 \quad-0.40560400 \quad 1.10196100
$$

$\mathrm{S}_{0}$ geometry of PT-2 in cyclohexane solvent, cam-b3lyp/tzvp/iefpcm, empiricaldispersion $=\mathrm{gd} 3 \mathrm{bj}$

Energy $=-963.763310$

\begin{tabular}{|c|c|c|c|}
\hline $\mathrm{C}$ & 5.10745600 & -1.38001800 & -0.00018900 \\
\hline $\mathrm{C}$ & 3.75155900 & -1.58513100 & -0.00046200 \\
\hline $\mathrm{C}$ & 2.89699800 & -0.47812100 & -0.00025000 \\
\hline $\mathrm{C}$ & 3.41281100 & 0.82828800 & 0.00020000 \\
\hline $\mathrm{C}$ & 4.80691300 & 1.00321600 & 0.00052400 \\
\hline $\mathrm{C}$ & 5.64149000 & -0.08278100 & 0.00034200 \\
\hline $\mathrm{H}$ & 5.77408300 & -2.23201200 & -0.00038000 \\
\hline $\mathrm{H}$ & 3.33783300 & -2.58517200 & -0.00086900 \\
\hline $\mathrm{C}$ & 2.48707900 & 1.90960500 & 0.00022600 \\
\hline $\mathrm{H}$ & 5.20728400 & 2.00893100 & 0.00092300 \\
\hline $\mathrm{H}$ & 6.71398100 & 0.05498800 & 0.00058800 \\
\hline $\mathrm{C}$ & 1.15034000 & 1.69310000 & -0.00013700 \\
\hline $\mathrm{C}$ & 0.64887800 & 0.36677200 & -0.00044900 \\
\hline $\mathrm{H}$ & 2.87349700 & 2.92127500 & 0.00060200 \\
\hline $\mathrm{H}$ & 0.44501700 & 2.51009500 & -0.00004000 \\
\hline $\mathrm{C}$ & -0.72224500 & 0.01169100 & -0.00023200 \\
\hline $\mathrm{C}$ & -1.88744300 & 0.79131600 & -0.00129900 \\
\hline $\mathrm{N}$ & -1.04889900 & -1.31035100 & 0.00069600 \\
\hline $\mathrm{C}$ & -2.87423600 & -0.16637700 & -0.00097700 \\
\hline $\mathrm{H}$ & -1.99940800 & 1.86157500 & -0.00238300 \\
\hline $\mathrm{H}$ & 1.11705900 & -1.56678400 & -0.00051500 \\
\hline $\mathrm{N}$ & -2.35096000 & -1.41879200 & 0.00038100 \\
\hline $\mathrm{N}$ & 1.53533700 & -0.63604700 & -0.00050400 \\
\hline $\mathrm{C}$ & -4.34306100 & 0.03099900 & 0.00000400 \\
\hline $\mathrm{F}$ & -4.65348300 & 1.34502100 & -0.01289700 \\
\hline $\mathrm{F}$ & -4.94649000 & -0.52131900 & -1.06997000 \\
\hline
\end{tabular}


F $\quad-4.94190500 \quad-0.49836100 \quad 1.08445200$

$\mathrm{S}_{1}$ geometry of QP-2 in cyclohexane solvent, cam-b3lyp/tzvp/iefpcm, empiricaldispersion $=\mathrm{gd} 3 \mathrm{bj}$

Energy $=-963.643073$

\begin{tabular}{|c|c|c|c|}
\hline $\mathrm{C}$ & 5.12124600 & -1.41367000 & 0.00103200 \\
\hline $\mathrm{C}$ & 3.75332700 & -1.59525700 & -0.00016800 \\
\hline $\mathrm{C}$ & 2.85267900 & -0.47901600 & -0.00046900 \\
\hline $\mathrm{C}$ & 3.42037000 & 0.85098300 & 0.00034600 \\
\hline $\mathrm{C}$ & 4.79183000 & 0.99082100 & 0.00137300 \\
\hline $\mathrm{C}$ & 5.64850100 & -0.13033000 & 0.00186400 \\
\hline $\mathrm{H}$ & 5.78065800 & -2.27110800 & 0.00133400 \\
\hline $\mathrm{H}$ & 3.31555400 & -2.58472400 & -0.00077300 \\
\hline $\mathrm{C}$ & 2.51388800 & 1.96153400 & -0.00044900 \\
\hline $\mathrm{H}$ & 5.22113400 & 1.98520900 & 0.00172600 \\
\hline $\mathrm{H}$ & 6.71981900 & 0.02054500 & 0.00282800 \\
\hline $\mathrm{C}$ & 1.16780300 & 1.71888000 & -0.00154900 \\
\hline $\mathrm{C}$ & 0.70181600 & 0.40051500 & -0.00177100 \\
\hline $\mathrm{H}$ & 2.90600900 & 2.96922500 & 0.00003000 \\
\hline $\mathrm{H}$ & 0.45753900 & 2.53491000 & -0.00189600 \\
\hline $\mathrm{C}$ & -0.67050500 & 0.06458200 & -0.00125800 \\
\hline $\mathrm{C}$ & -1.86930800 & 0.79625300 & -0.00070600 \\
\hline $\mathrm{N}$ & -1.05745500 & -1.24772100 & -0.00122100 \\
\hline $\mathrm{C}$ & -2.85920400 & -0.17210600 & -0.00028600 \\
\hline $\mathrm{H}$ & -1.99195700 & 1.86422100 & -0.00057600 \\
\hline $\mathrm{H}$ & -0.41317900 & -2.02603400 & -0.00168400 \\
\hline $\mathrm{N}$ & -2.36600900 & -1.41393400 & -0.00058400 \\
\hline $\mathrm{N}$ & 1.55101500 & -0.70762500 & -0.00171600 \\
\hline $\mathrm{C}$ & -4.33599400 & 0.03214000 & 0.00077200 \\
\hline $\mathrm{F}$ & -4.62887400 & 1.34418600 & -0.00145800 \\
\hline $\mathrm{F}$ & -4.92594100 & $\begin{array}{l}-0.51085500 \\
\text { s10 }\end{array}$ & -1.07553200 \\
\hline
\end{tabular}




$$
\text { F } \quad-4.92375500 \quad-0.50658200 \quad 1.08046400
$$

$\mathrm{S}_{1}$ geometry of PT-2 in cyclohexane solvent, cam-b3lyp/tzvp/iefpcm, empiricaldispersion $=\mathrm{gd} 3 \mathrm{bj}$

Energy $=-963.648550$

\begin{tabular}{|c|c|c|c|}
\hline $\mathrm{C}$ & 5.05916000 & -1.45594700 & 0.00038300 \\
\hline $\mathrm{C}$ & 3.69100400 & -1.60746900 & 0.00016200 \\
\hline $\mathrm{C}$ & 2.86653700 & -0.47870100 & -0.00002300 \\
\hline $\mathrm{C}$ & 3.42588300 & 0.84067200 & 0.00002400 \\
\hline $\mathrm{C}$ & 4.82981900 & 0.94679800 & 0.00025500 \\
\hline $\mathrm{C}$ & 5.62656800 & -0.17324200 & 0.00042900 \\
\hline $\mathrm{H}$ & 5.69736900 & -2.32893500 & 0.00052100 \\
\hline $\mathrm{H}$ & 3.24036100 & -2.59204100 & 0.00012200 \\
\hline $\mathrm{C}$ & 2.55759600 & 1.95193400 & -0.00015800 \\
\hline $\mathrm{H}$ & 5.27228800 & 1.93452400 & 0.00029100 \\
\hline $\mathrm{H}$ & 6.70333200 & -0.06658200 & 0.00060700 \\
\hline $\mathrm{C}$ & 1.17396900 & 1.75499600 & -0.00031100 \\
\hline $\mathrm{C}$ & 0.65805300 & 0.48496900 & -0.00033100 \\
\hline $\mathrm{H}$ & 2.97086400 & 2.94980500 & -0.00015200 \\
\hline $\mathrm{H}$ & 0.49498400 & 2.59522500 & -0.00040000 \\
\hline $\mathrm{C}$ & -0.72380700 & 0.10351400 & -0.00029100 \\
\hline $\mathrm{C}$ & -1.91571200 & 0.84016500 & 0.00009100 \\
\hline $\mathrm{N}$ & -1.02605400 & -1.26336600 & -0.00052300 \\
\hline $\mathrm{C}$ & -2.87170100 & -0.13902300 & -0.00004500 \\
\hline $\mathrm{H}$ & -2.05223900 & 1.90691400 & 0.00038900 \\
\hline $\mathrm{H}$ & 1.05969500 & -1.51230300 & -0.00031800 \\
\hline $\mathrm{N}$ & -2.30432900 & -1.40436400 & -0.00040000 \\
\hline $\mathrm{N}$ & 1.51146900 & -0.60142500 & -0.00027300 \\
\hline $\mathrm{C}$ & -4.35128700 & 0.00413000 & 0.00013900 \\
\hline $\mathrm{F}$ & -4.70616400 & 1.30137800 & 0.00065400 \\
\hline $\mathrm{F}$ & -4.91427900 & $\begin{array}{l}-0.56357400 \\
\text { S11 }\end{array}$ & -1.07869600 \\
\hline
\end{tabular}




$$
\text { F } \quad-4.91408600 \quad-0.56439100 \quad 1.07863900
$$

$\mathrm{S}_{1}$ geometry of TICT-2 in cyclohexane solvent, cam-b3lyp/tzvp/iefpcm, empiricaldispersion $=\mathrm{gd} 3 \mathrm{bj}$

Energy $=-963.649612$

\begin{tabular}{|c|c|c|c|}
\hline $\mathrm{C}$ & 4.99041100 & -1.61879400 & -0.05099000 \\
\hline $\mathrm{C}$ & 3.61900500 & -1.69050600 & 0.12299200 \\
\hline $\mathrm{C}$ & 2.85021000 & -0.53588500 & 0.05773700 \\
\hline $\mathrm{C}$ & 3.45034400 & 0.71697100 & -0.18298900 \\
\hline $\mathrm{C}$ & 4.83535600 & 0.75505100 & -0.35726100 \\
\hline $\mathrm{C}$ & 5.59990700 & -0.39467100 & -0.29284300 \\
\hline $\mathrm{H}$ & 5.58347000 & -2.52171600 & 0.00313600 \\
\hline $\mathrm{H}$ & 3.13978900 & -2.64379300 & 0.31029500 \\
\hline $\mathrm{C}$ & 2.61636800 & 1.88644600 & -0.23222800 \\
\hline $\mathrm{H}$ & 5.30688800 & 1.71212800 & -0.54223500 \\
\hline $\mathrm{H}$ & 6.67155300 & -0.33986100 & -0.42900900 \\
\hline $\mathrm{C}$ & 1.26317700 & 1.76999200 & -0.04771100 \\
\hline $\mathrm{C}$ & 0.67609600 & 0.53836900 & 0.18852400 \\
\hline $\mathrm{H}$ & 3.06867100 & 2.85071500 & -0.41091300 \\
\hline $\mathrm{H}$ & 0.62649300 & 2.64291000 & -0.08263800 \\
\hline $\mathrm{C}$ & -0.75236800 & 0.34357600 & 0.35083900 \\
\hline $\mathrm{C}$ & -1.74890800 & 0.09876300 & -0.62830400 \\
\hline $\mathrm{N}$ & -1.36325600 & 0.35555300 & 1.62543700 \\
\hline $\mathrm{C}$ & -2.87797300 & -0.00996300 & 0.09561700 \\
\hline $\mathrm{H}$ & -1.61518800 & 0.03088600 & -1.69269300 \\
\hline $\mathrm{H}$ & 1.06561000 & -1.47613400 & 0.48022500 \\
\hline $\mathrm{N}$ & -2.60362300 & 0.14692500 & 1.48680400 \\
\hline $\mathrm{N}$ & 1.47351800 & -0.59266000 & 0.22595200 \\
\hline $\mathrm{C}$ & -4.27856800 & -0.25936800 & -0.34081100 \\
\hline $\mathrm{F}$ & -4.35330000 & -0.35649400 & -1.67626900 \\
\hline $\mathrm{F}$ & -4.75737600 & $\begin{array}{l}-1.39865800 \\
\text { S12 }\end{array}$ & 0.17767400 \\
\hline
\end{tabular}




$$
\text { F } \quad-5.09511100 \quad 0.73029200 \quad 0.04315600
$$

$\mathrm{S}_{1}$ geometry of Trans-2 in cyclohexane solvent, cam-b3lyp/tzvp/iefpcm, empiricaldispersion $=\mathrm{gd} 3 \mathrm{bj}$

Energy $=-963.640591$

\begin{tabular}{|c|c|c|c|}
\hline $\mathrm{C}$ & 4.72587200 & -1.86996900 & 0.16111900 \\
\hline $\mathrm{C}$ & 3.35060900 & -1.79833800 & 0.16099900 \\
\hline $\mathrm{C}$ & 2.71977600 & -0.56157700 & 0.01879100 \\
\hline $\mathrm{C}$ & 3.48281500 & 0.64226700 & -0.10425800 \\
\hline $\mathrm{C}$ & 4.88838200 & 0.52153800 & -0.09394100 \\
\hline $\mathrm{C}$ & 5.49404400 & -0.70331200 & 0.03257000 \\
\hline $\mathrm{H}$ & 5.21392900 & -2.82970800 & 0.26230000 \\
\hline $\mathrm{H}$ & 2.75174300 & -2.69530600 & 0.26131600 \\
\hline $\mathrm{C}$ & 2.80593300 & 1.87082600 & -0.21422000 \\
\hline $\mathrm{H}$ & 5.48235900 & 1.42164300 & -0.18424000 \\
\hline $\mathrm{H}$ & 6.57376700 & -0.77246300 & 0.03652700 \\
\hline $\mathrm{C}$ & 1.40806900 & 1.90352200 & -0.17972200 \\
\hline $\mathrm{C}$ & 0.68847300 & 0.74373800 & -0.06033400 \\
\hline $\mathrm{H}$ & 3.37324700 & 2.78610600 & -0.30003200 \\
\hline $\mathrm{H}$ & 0.86345400 & 2.83427200 & -0.22937400 \\
\hline $\mathrm{C}$ & -0.74535500 & 0.66976200 & 0.04510800 \\
\hline $\mathrm{C}$ & -1.61159200 & -0.42118600 & -0.22006800 \\
\hline $\mathrm{N}$ & -1.49324800 & 1.76774400 & 0.43459600 \\
\hline $\mathrm{C}$ & -2.84090800 & 0.10742000 & 0.03384800 \\
\hline $\mathrm{H}$ & -1.37515700 & -1.41166000 & -0.56912100 \\
\hline $\mathrm{H}$ & 0.81366100 & -1.30323000 & 0.15465200 \\
\hline $\mathrm{N}$ & -2.74023400 & 1.43950700 & 0.41155500 \\
\hline $\mathrm{N}$ & 1.35513900 & -0.46742000 & -0.01214000 \\
\hline $\mathrm{C}$ & -4.17347500 & -0.53881600 & -0.05715100 \\
\hline $\mathrm{F}$ & -4.05368900 & -1.83041500 & -0.41930000 \\
\hline $\mathrm{F}$ & -4.83251000 & $\begin{array}{l}-0.51394100 \\
\text { S13 }\end{array}$ & 1.11357100 \\
\hline
\end{tabular}


$\begin{array}{llll}\text { F } & -4.96985200 & 0.05505300 & -0.96166500\end{array}$

$\mathrm{S}_{0}$ geometry of QP-3 in cyclohexane solvent, cam-b3lyp/tzvp/iefpcm, empiricaldispersion $=\mathrm{gd} 3 \mathrm{bj}$

Energy=-963.779422

\begin{tabular}{|c|c|c|c|}
\hline $\mathrm{C}$ & 4.02740300 & 2.21441300 & 0.04983600 \\
\hline $\mathrm{C}$ & 4.39465300 & 0.91757700 & -0.15056600 \\
\hline $\mathrm{C}$ & 3.42581700 & -0.11216300 & -0.13875500 \\
\hline $\mathrm{C}$ & 2.06141000 & 0.21804700 & 0.05419300 \\
\hline $\mathrm{C}$ & 1.71906700 & 1.56975100 & 0.29415600 \\
\hline $\mathrm{C}$ & 2.67760700 & 2.54034900 & 0.28972900 \\
\hline $\mathrm{H}$ & 4.80836500 & -1.75462900 & -0.42615700 \\
\hline $\mathrm{H}$ & 4.77335800 & 2.99844500 & 0.04359500 \\
\hline $\mathrm{H}$ & 5.43276900 & 0.65467500 & -0.31010600 \\
\hline $\mathrm{C}$ & 3.77396200 & -1.47150900 & -0.28228100 \\
\hline $\mathrm{C}$ & 1.12434100 & -0.85833400 & 0.02334600 \\
\hline $\mathrm{H}$ & 0.69710900 & 1.83437800 & 0.51398500 \\
\hline $\mathrm{H}$ & 2.40304000 & 3.56900600 & 0.48256800 \\
\hline $\mathrm{C}$ & 2.79743400 & -2.41697500 & -0.21749300 \\
\hline $\mathrm{H}$ & 3.03016400 & -3.47162800 & -0.29294800 \\
\hline $\mathrm{C}$ & -0.33124500 & -0.66639300 & 0.10873500 \\
\hline $\mathrm{C}$ & -1.20855900 & 0.34338200 & -0.23110200 \\
\hline $\mathrm{N}$ & -1.10611100 & -1.68909800 & 0.54067000 \\
\hline $\mathrm{C}$ & -2.47317800 & -0.19280300 & 0.04313500 \\
\hline $\mathrm{H}$ & -0.99294400 & 1.30561900 & -0.65570200 \\
\hline $\mathrm{C}$ & -3.79815900 & 0.46569000 & -0.13240900 \\
\hline $\mathrm{F}$ & -3.64659400 & 1.68740500 & -0.67587100 \\
\hline $\mathrm{F}$ & -4.61571400 & -0.23034400 & -0.93716000 \\
\hline $\mathrm{F}$ & -4.44774600 & 0.62870400 & 1.03258700 \\
\hline $\mathrm{N}$ & -2.40351200 & -1.42596700 & 0.51068700 \\
\hline $\mathrm{N}$ & 1.48976000 & $\begin{array}{l}-2.11529800 \\
\text { S14 }\end{array}$ & -0.07983300 \\
\hline
\end{tabular}


$\mathrm{S}_{1}$ geometry of QP-3 in cyclohexane solvent, cam-b3lyp/tzvp/iefpcm, empiricaldispersion $=\mathrm{gd} 3 \mathrm{bj}$

Energy=-963.645394

\begin{tabular}{|c|c|c|c|}
\hline $\mathrm{C}$ & 3.94769000 & 2.25037400 & -0.12834600 \\
\hline $\mathrm{C}$ & 4.25350000 & 0.94067300 & -0.49391600 \\
\hline $\mathrm{C}$ & 3.33166100 & -0.09687400 & -0.29784800 \\
\hline $\mathrm{C}$ & 2.04829400 & 0.21468800 & 0.25485300 \\
\hline $\mathrm{C}$ & 1.78532700 & 1.51167100 & 0.65429300 \\
\hline $\mathrm{C}$ & 2.72841700 & 2.53446600 & 0.44813900 \\
\hline $\mathrm{H}$ & 4.59122000 & -1.73054700 & -0.95060400 \\
\hline $\mathrm{H}$ & 4.67087400 & 3.03780800 & -0.29209700 \\
\hline $\mathrm{H}$ & 5.20841600 & 0.71366500 & -0.95113800 \\
\hline $\mathrm{C}$ & 3.61228200 & -1.44419500 & -0.58943500 \\
\hline $\mathrm{C}$ & 1.10136600 & -0.87271000 & 0.29582100 \\
\hline $\mathrm{H}$ & 0.85587700 & 1.74324300 & 1.15516300 \\
\hline $\mathrm{H}$ & 2.49186900 & 3.54381600 & 0.75743900 \\
\hline $\mathrm{C}$ & 2.71471200 & -2.44441600 & -0.15661500 \\
\hline $\mathrm{H}$ & 3.04138500 & -3.47891400 & -0.13556800 \\
\hline $\mathrm{C}$ & -0.29937400 & -0.70719800 & 0.23433100 \\
\hline $\mathrm{C}$ & -1.15930600 & 0.37320200 & -0.01411800 \\
\hline $\mathrm{N}$ & -1.12969900 & -1.78647300 & 0.38605300 \\
\hline $\mathrm{C}$ & -2.43146600 & -0.18580200 & 0.01726200 \\
\hline $\mathrm{H}$ & -0.89809600 & 1.39157000 & -0.23234000 \\
\hline $\mathrm{C}$ & -3.73412700 & 0.50897300 & -0.18820400 \\
\hline $\mathrm{F}$ & -3.53579500 & 1.81329700 & -0.45043300 \\
\hline $\mathrm{F}$ & -4.43067200 & -0.00268600 & -1.21505500 \\
\hline $\mathrm{F}$ & -4.52762000 & 0.43742800 & 0.89307200 \\
\hline $\mathrm{N}$ & -2.41421700 & -1.49134300 & 0.26368600 \\
\hline $\mathrm{N}$ & 1.53163200 & $\begin{array}{l}-2.18253800 \\
\text { S15 }\end{array}$ & 0.32216200 \\
\hline
\end{tabular}


